\title{
TEORÍA DEL CAOS Y ESTRATEGIA EMPRESARIAL
}

\author{
CHAOS THEORY AND BUSINESS STRATEGY \\ TEORIA DO CAOS E ESTRATÉGIA DE NEGOCIOS
}

MARTÍNEZ MONCALEANO_Carlos Javier

\begin{abstract}
Estudiante de la Maestría en Estudios Interdisciplinarios de la Complejidad, Universidad Surcolombiana. Administrador de Empresas de la Universidad Surcolombiana. Email: cartan1991@hotmail.com, Colombia.
\end{abstract}

Recibido: 19 de febrero de 2018 Aprobación definitiva: 5 de junio de 2018

DOI: http://dx.doi.org/10.22267/rtend.181901.94

\begin{abstract}
RESUMEN
Este artículo propone un acercamiento de la teoría del caos a los modelos estratégicos empresariales. En primer lugar, se exponen algunos de los elementos fundamentales del caos, así, como de las ciencias de la complejidad en general, seguidamente, se plantean las nociones teóricas de la estrategia empresarial, considerando la enorme cantidad de variables que inciden en la organización y que condicionan sus diferentes atractores.
\end{abstract}

Palabras Clave: Teoría del Caos, Estrategia Empresarial, Organizaciones.

\section{JEL: M10, M21, M50}

\section{ABSTRACT}

This article proposes an approach of chaos theory to strategic business models. In first place, some of the fundamental elements of chaos and sciences of complexity in general are exposed. Then, the theoretical notions of the business strategy are considered, taking in consideration 
the enormous number of variables that affect the organization and its different attractors.

Keywords: Chaos Theory, Business Strategy, Organizations. JEL: M10, M21, M50

\section{RESUMO}

Este artigo propõe uma abordagem da teoria do caos a modelos de negócios estratégicos, en primeiro lugar estão expostos alguns elementos fundamentais do caos como de as ciências da complexidade em geral. São consideradas as noções teóricas da estratégia de negócios, considerando o grande número de variáveis que afetam a organização e que condicionam seus diferentes atratores.

Palavras-chave: Teoria do Caos, Estratégia de Negócios, Organizações.

JEL: M10, M21, M50

\section{INTRODUCCIÓN}

No hay lugar a dudas que las organizaciones empresariales, han sido, históricamente, un elemento fundamental para el crecimiento económico y el desarrollo social del ser humano. Hay que señalar que las empresas, son las responsables de producir los bienes y servicios que satisfacen diversas necesidades de las personas y que, además, son los principales motores de las economías. Jones (2008), señala que las organizaciones son "una herramienta que utilizan las personas para coordinar sus acciones con el fin de obtener algo que desean o valoran, es decir, lograr sus metas...una organización es la respuesta a alguna necesidad humana y el medio de satisfacerla" (p.2).

Desde la aparición de la denominada escuela de administración científica, basada en los aportes de Frederick Taylor, la cual enfatiza en el estudio de tiempos y movimientos por medio de la observación directa al operario y de la escuela clásica de Henri Fayol y Henry Ford, centrada en los procesos administrativos, estando estos compuestos por los subprocesos de planeación, organización, dirección y control, han aparecido diversas escuelas y teorías administrativas y organizacionales, 
como son la escuela burocrática, la escuela de la calidad total o la administración contingencial. Esto dio lugar a que la organización empresarial pasara de ser concebida como un sistema cerrado, de casi nula interacción con el medio y centrada en procesos de producción per se, a un sistema abierto, dinámico, no lineal, que obedeciendo su interrelación y dependencia con el entorno, debe adaptarse a los constantes cambios del mismo.

Estos cambios en la concepción de la organización empresarial, han permitido una importante aplicación de la teoría del caos $y$, en general, de las denominadas ciencias de la complejidad a los procesos de planificación y dirección de empresas. Se entiende, por tanto, que herramientas estratégicas que consideren los fenómenos no lineales, la incertidumbre, los cambios súbitos y la auto organización, pueden ser de enorme ayuda en la propuesta y formulación de los aspectos estratégicos y teleológicos de la organización empresarial.

\section{LAS CIENCIAS DE LA COMPLEJIDAD}

Maldonado y Gómez (2010), proponen que las ciencias de la complejidad son:

Un grupo de ciencias -que por tanto contienen numerosas teorías, una diversidad de modelos explicativos, una gama amplia de conceptos, en fin, una pluralidad de métodos y lógicas- cuyo tema de base es, para decirlo en términos genéricos: ¿Por qué las cosas son o se vuelven complejas? ¿Qué es, al fin y al cabo, "complejidad"? (P.12).

Considerando, además, que no existe una definición concreta y única de lo que es complejidad.

Se puede decir, por tanto, que las ciencias de la complejidad, estudian, precisamente, los sistemas complejos, es decir, los sistemas abiertos, no lineales, auto organizados y alejados del equilibrio.

Se debe aclarar, además, que todas las ciencias de la complejidad, nacieron de las denominadas ciencias básicas - matemáticas, física, estadística etc. - y que, con el devenir de los años y con la aparición 
de diversos paradigmas científicos, encontraron su espacio en las ciencias y disciplinas sociales y humanas. Las corrientes dominantes del estudio de los sistemas complejos son, asimismo, interdisciplinarias y transdisciplinarias.

Maldonado y Gómez (2010) dicen que, cronológicamente, las ciencias de la complejidad son: La termodinámica del no equilibrio, la teoría del caos, la geometría fractal, la teoría de catástrofes, las lógicas no clásicas y las redes complejas. Como se puede observar en la tabla, se exponen las principales características y autores de las ciencias de la complejidad:

\section{Tabla 1}

Las ciencias de la complejidad y sus áreas de estudio

\begin{tabular}{|c|c|c|c|}
\hline $\begin{array}{l}\text { Ciencia de la } \\
\text { complejidad }\end{array}$ & Área de estudio & Inicios & $\begin{array}{c}\text { Autores } \\
\text { destacados }\end{array}$ \\
\hline $\begin{array}{l}\text { Termodinámica } \\
\text { del no Equilibrio }\end{array}$ & $\begin{array}{l}\text { Los sistemas alejados del } \\
\text { equilibrio termodinámico y } \\
\text { los procesos irreversibles. }\end{array}$ & $\begin{array}{l}\text { Principios de la } \\
\text { década de los } \\
\text { cuarenta. } \\
\end{array}$ & $\begin{array}{l}\text { Ilya prigogine } \\
\text { Constantino } \\
\text { Tsallis } \\
\end{array}$ \\
\hline Teoría del Caos & $\begin{array}{l}\text { Los sistemas complejos } \\
\text { sensibles a condiciones } \\
\text { iniciales. }\end{array}$ & $\begin{array}{l}\text { Finales de la } \\
\text { década de los } \\
\text { cincuenta. }\end{array}$ & $\begin{array}{l}\text { Edward Lorenz } \\
\text { Stephen Kellert }\end{array}$ \\
\hline $\begin{array}{l}\text { Teoría de } \\
\text { Catástrofes }\end{array}$ & $\begin{array}{l}\text { Cambios súbitos e } \\
\text { irreversibles en sistemas } \\
\text { estructuralmente estables }\end{array}$ & $\begin{array}{l}\text { Finales de la } \\
\text { década de los } \\
\text { cincuenta }\end{array}$ & René Thom \\
\hline $\begin{array}{l}\text { Geometría } \\
\text { Fractal }\end{array}$ & $\begin{array}{l}\text { Los fractales. Figuras } \\
\text { autosemejantes de } \\
\text { dimensión no entera. }\end{array}$ & $\begin{array}{l}\text { Década de los } \\
\text { setenta. }\end{array}$ & $\begin{array}{l}\text { Benoit } \\
\text { Mandelbrot }\end{array}$ \\
\hline $\begin{array}{l}\text { Lógicas no } \\
\text { Clásicas }\end{array}$ & $\begin{array}{l}\text { La lógica difusa, la lógica } \\
\text { paraconsistente, la lógica } \\
\text { cuántica, la lógica libre } \\
\text { etc. }\end{array}$ & $\begin{array}{l}\text { A lo largo del siglo } \\
X X \text {, con grandes } \\
\text { avances en los } \\
\text { años noventa. }\end{array}$ & $\begin{array}{l}\text { Jean Louis } \\
\text { Gardes } \\
\text { Lofti Zadeh } \\
\text { Francisco Miró }\end{array}$ \\
\hline $\begin{array}{l}\text { Redes } \\
\text { Complejas }\end{array}$ & $\begin{array}{l}\text { Redes que presentan } \\
\text { ciertas características } \\
\text { estadísticas y topológicas. }\end{array}$ & $\begin{array}{l}\text { Inicios del siglo } \\
\text { XXI. }\end{array}$ & $\begin{array}{l}\text { Lászlo Barabasi } \\
\text { Steve Strogatz }\end{array}$ \\
\hline
\end{tabular}

Fuente: Maldonado y Gomez (2010) 
Estas ciencias no solo son una respuesta a las ciencias tradicionales, y a su enfoque cerrado e inductivo, sino, configuran un mundo de posibilidades de desarrollo científico y epistemológico. Esto se complementa con los aportes de Rodriguez y Aguirre (2011) quienes proponen que la complejidad:

Constituye una perspectiva novedosa y marginal en la ciencia contemporánea; su carácter de novedad radica en que el estudio de la complejidad implica, en buena medida, un quiebre o discontinuidad en la historia de la ciencia o, más precisamente dicho, en la racionalidad científica occidental. La complejidad introduce, en el terreno de las ciencias, una racionalidad postclásica que habilita e incorpora problemas ignorados o vedados por el pensamiento científico moderno. Estos problemas involucran, en un sentido no exhaustivo, cuestiones relativas al desorden, el caos, la no-linealidad, el no-equilibro, la indecibilidad, la incertidumbre, la contradicción, el azar, la temporalidad, la emergencia, la auto-organizacion. (p.2)

\section{LA TEORÍA DEL CAOS}

La teoría del caos es un campo de estudio fundamental dentro de las ciencias de la complejidad, siendo, a lo largo de los años y desde que el climatólogo Edward Lorenz la propuso formalmente a principios de los años 60 , un paradigma, que ha supuesto importantes y profundos cambios en el estudio de diversos fenómenos. Con el caos, se consagra, por tanto, la idea de la no linealidad en la ciencia y, en general, se establece la enorme dificultad de predecir la evolución de determinados sistemas.

En términos generales, la teoría del caos estudia la sensibilidad a condiciones iniciales de algunos sistemas, es decir, de aquellos sistemas en los que un pequeño cambio, puede generar grandes consecuencias. Matemáticamente, estos cambios siguen una dinámica no lineal (Cambel, 1993).

La teoría del caos es entonces la ciencia del proceso, el intento del conocimiento, de lo que va a suceder, no de lo que es o de lo que permanecerá. Con ella lo desconocido se interpreta desde un punto de vista global, que se ve afectado en el tiempo. Hablar de caos es hablar 
de procesos irreversibles, es decir de procesos que dejan huella en el tiempo. Hablar del caos, es asimismo, considerar que desde estados de desorden se llega a estados de orden (Ferrari, 2003).

Es importante aclarar que, a grandes rasgos, los sistemas y fenómenos caóticos, cuentan con unas determinadas características.

En primer lugar, tienen una condición de sistema abierto. En este sentido, Cambel (1993) propone que un sistema abierto tiene "muros permeables y por tanto la materia, energía e información o entropía pueden cruzar hacia cualquier dirección" (p.42), propone, asimismo, que como experimentan procesos irreversibles "al ser rastreados hacia atrás, algún tipo de evidencia va a ser encontrada" (p.54).

Por otra parte, los fenómenos caóticos son no lineales, es decir que sus efectos no son proporcionales a sus causas (Pidal, 2009). Es importante aclarar, además, que los denominados sistemas "vivos" son caóticos. Esto, se complementa con Chaparro (2008) quien expone que:

La realimentación da lugar al comportamiento no lineal. Un sistema que se describa por medio de ecuaciones no lineales no puede ser sujeto del principio de superposición, la acción de cada una de sus partes no puede ser separada, el sistema debe ser considerado en su totalidad. La comprensión de que existen mecanismos de realimentación entre las variables de un sistema que dan lugar a comportamientos no lineales es el punto de partida para entender la conducta 'caótica' y compleja de muchos fenómenos naturales y sociales (p. 201).

Finalmente, los sistemas caóticos evolucionan dado un atractor extraño y por tanto el sistema presenta un comportamiento aperiódico a largo plazo; existen trayectorias que con el paso del tiempo no convergen a órbitas periódicas, cuasiperiódicas, o puntos fijos, y exhiben una dependencia sensible a pequeñas variaciones en las condiciones iniciales, lo que significa que dos trayectorias cercanas, a medida que transcurre el tiempo se separan de forma exponencial (Moreno, Parra, Huérfano \& Suárez, 2016) La siguiente tabla describe, entonces, las diferencias principales entre el enfoque tradicional y el enfoque del caos. 


\section{Tabla 2}

Enfoque tradicional y enfoque del caos.

\begin{tabular}{|l|l|}
\hline \multicolumn{1}{|c|}{ Enfoque Tradicional } & \multicolumn{1}{c|}{ Enfoque del caos } \\
\hline $\begin{array}{l}\text { Es posible predecir el comportamiento } \\
\text { de cualquier estado futuro del sistema } \\
\text { a través de una ecuación simple causa- } \\
\text { efecto. }\end{array}$ & $\begin{array}{l}\text { No hay proporcionalidad en la relación } \\
\text { causa-efecto. El futuro es incierto } \\
\text { impredecible, la evolución del sistema no } \\
\text { ocurre de manera continua. }\end{array}$ \\
\hline El todo es la suma de las partes. & $\begin{array}{l}\text { El todo complejo está hecho de infinitas } \\
\text { iteraciones de un patrón simple que es } \\
\text { repetido en escalas diferentes. }\end{array}$ \\
\hline $\begin{array}{l}\text { El caos es sinónimo de desorden y puede } \\
\text { evitarse controlando el sistema todo lo } \\
\text { que sea posible. }\end{array}$ & $\begin{array}{l}\text { Hay una relación estrecha entre el caos } \\
\text { y el orden, tanto que uno conduce al otro } \\
\text { siguiendo un proceso dinámico. No se } \\
\text { trata de evitar el caos, se trata de usarlo } \\
\text { para autoorganizar su sistema a través } \\
\text { de un 'atractor' }\end{array}$ \\
\hline $\begin{array}{l}\text { El sistema no cambia de manera } \\
\text { repentina, si lo hace se debe a algún } \\
\text { error que no ha sido bien controlado. }\end{array}$ & $\begin{array}{l}\text { Una pequeña perturbación puede causar } \\
\text { repentinamente cambios explosivos } \\
\text { dentro del Sistema. }\end{array}$ \\
\hline $\begin{array}{l}\text { Un elemento no puede pertenecer al } \\
\text { mismo tiempo a un conjunto y a su } \\
\text { complemento. }\end{array}$ & $\begin{array}{l}\text { La relación entre los elementos y los } \\
\text { conjuntos no es solo sí o no, es un asunto } \\
\text { de más o menos. }\end{array}$ \\
\hline
\end{tabular}

Fuente: Lisak ( en Salazar 2017)

\section{LA ESTRATEGIA EMPRESARIAL Y EL CAOS}

A la hora de formular una estrategia empresarial se debe tomar en consideración, la gran cantidad de variables que inciden en la evolución de la empresa y, por tanto, se debe dar lugar a acciones que permitan "navegar" entre toda la turbulencia e incertidumbre que rodea la organización empresarial.

Es entonces que Ferrari (2003:1) señala las características inherentes de la estrategia empresarial.

a) la imposibilidad de que su concepción sea lineal,

b) la complejidad de los aspectos a considerar, 
c) la necesidad de conocer las profundas interrelaciones entre las variables,

d) la creciente dificultad de su concepción considerando que cada vez hay más actores a considerar, nuevas asociaciones entre empresas, nuevas soluciones y nuevos servicios.

Levy (1994) considera la enorme dificultad de realizar planificación a largo plazo, debido a la extrema sensibilidad a condiciones iniciales.

La planificación a largo plazo de un sistema caótico no es solo difícil, si no, esencialmente imposible... además que estrechar el foco en los mercados y productos principales de la empresa, podría reducir la capacidad de la adaptación y de flexibilidad al cambio de la organización (p.170).

Sin embargo, Levy (1994) aclara que se puede realizar planificación y predicción de patrones a corto plazo ya que:

Hay también un enorme grado de orden en los sistemas caóticos, La planificación a corto plazo es posible en un sistema determinístico dadas unas condiciones de tiempo " $\mathrm{t}$ ", se puede calcular condiciones de tiempo " $t+1$ ". Un modelo de simulación de sistema complejo cuidadosamente construido, dotado de unas condiciones iniciales precisas puede ser de gran ayuda en la planificación de, por lo menos, varios periodos de tiempo (p. 171)

En este sentido, el concepto de caos en la organización representa la evolución natural que contiene incertidumbre en un ambiente turbulento ya que, desde este enfoque, como se propuso antes, se explica la relación entre el orden y el desorden; el caos es de hecho un aspecto necesario para lograr una constante renovación y dinamismo en la empresa (Pidal, 2009).

Complementando estas ideas, se debe destacar que las empresas evolucionan dados diversos atractores. La naturaleza de estos atractores es variada y, en muchas ocasiones, se pueden tornar contrarios a los propios objetivos estratégicos de la organización. 
Según Cornejo (2004), los atractores de la empresa son: la visión de la organización, la misión, el grupo de poder y su producto o servicio. Cornejo aclara, que:

Estos atractores son aquellos marcos de referencia que permiten la dinámica compleja de la organización en sus diferentes dimensiones, pero por otro lado establecen ciertos límites para el desempeño de las variables. Dentro de esos límites las variables están sujetas a una dinámica bastante compleja, y el conjunto de interacciones dinámicas entre esas variables en ese estado. (p.4).

Como se puede apreciar, existen atractores formales, formulados de manera planificada y, asímismo, hay atractores más complejos, que obedecen a factores alejados del control de la gerencia. Desde un estado de desorden la gerencia puede guiar la dinámica caótica para alcanzar los objetivos deseados (Salazar, 2017).

Cambios súbitos e imprevistos son siempre posibles. La estrategia empresarial debe estar diseñada, entonces, dada una flexibilidad y adaptabilidad que responda a estas fluctuaciones. Es menester para la alta gerencia proponer y consolidar estrategias adecuadas y verosímiles tomando en consideración las múltiples variables implicadas en el contexto de la organización. Asímismo, se debe tomar en cuenta que no es posible controlar todos los atractores inmersos y si bien, es necesario formular un diseño teleológico pertinente y desarrollar una estructura organizacional que se acomode a las diversas necesidades, hay que entender que las organizaciones empresariales no evolucionan recorriendo un camino unidireccional y en línea recta, por el contrario, lo hacen siguiendo puntos dispersos, en un principio predecibles, que posteriormente, se vuelven aperiódicos y finalmente caóticos, es por ello que la flexibilidad, la creatividad y la adaptabilidad deben ser características intrínsecas de la estrategia empresarial.

En este sentido, se propone la siguiente comparación entre el enfoque "clásico" de estrategia empresarial y el enfoque estratégico desde la teoría del caos: 
Tabla 3

Enfoque de Estrategia Empresarial clásico y Enfoque desde la Teoría del Caos

\begin{tabular}{|l|l|l|}
\hline \multicolumn{1}{|c|}{ Aspecto } & \multicolumn{1}{|c|}{ Enfoque Clásico } & \multicolumn{1}{c|}{$\begin{array}{c}\text { Enfoque desde la Teoría del } \\
\text { Caos }\end{array}$} \\
\hline $\begin{array}{l}\text { Concepción de la } \\
\text { empresa }\end{array}$ & $\begin{array}{l}\text { Sistema lineal con escasa } \\
\text { interacción con el medio }\end{array}$ & $\begin{array}{l}\text { Sistema no lineal con enorme } \\
\text { interacción con el medio. }\end{array}$ \\
\hline $\begin{array}{l}\text { Formulación de } \\
\text { estrategias }\end{array}$ & Rígidas y sistemáticas & Adaptativas y dinámicas \\
\hline $\begin{array}{l}\text { Énfasis } \\
\text { estratégico }\end{array}$ & Cumplimiento de objetivos & $\begin{array}{l}\text { Adaptación constante a los } \\
\text { cambios internos y externos de } \\
\text { la empresa. }\end{array}$ \\
\hline
\end{tabular}

Fuente: Elaboración propia.

\section{CONCLUSIONES}

En las últimas décadas, la teoría del caos ha supuesto un cambio paradigmático en diversas ciencias naturales y sociales. La idea de que, en determinados casos no exista una relación lineal de causa y efecto o, que es lo mismo, que un cambio pequeño en una condición inicial de un sistema pueda generar grandes consecuencias en la evolución del mismo, ha permitido el surgimiento tanto de modelos teóricos y conceptuales en el estudio de diversos fenómenos, como de modelos matemáticos y computacionales que han ayudado a abordar el comportamiento de dichos fenómenos.

Las empresas, por antonomasia, viven inmersas en la incertidumbre debido a las distintas variables que hacen que evolucionen dados diversos atractores, por tanto, la gerencia debe hacer una gestión del caos con el fin de generar un mayor dinamismo dentro del sistema empresarial.

Un modelo estratégico empresarial debe ser flexible y se debe adaptar a distintas condiciones; las estrategias se deben configurar como un atractor que permita a la empresa alcanzar sus objetivos estratégicos. 


\section{REFERENCIAS}

(1) Cambel A.B. (1993) Applied chaos theory -A paradigm for complexity, Academic Press, Inc., New York, Harcourt Brace Jovanovich.

(2) Chaparro Guevara G. (2008) No linealidad, Complejidad y Sistemas Sociales. Disponible en: http://virajes.ucaldas.edu.co/downloads/virajes10_8.pdf

(3) Cornejo Alvarez A. (2004) Complejidad Y Caos: Guía Para La Administración Del Siglo XXI.

(4) Ferrari, C. A. (2003). La Teoría del Caos y la estrategia en los sistemas complejos. Técnica administrativa.

(5) Jones G. R (2008). Teoría Organizacional: Diseño y Cambio en las Organizaciones. Quinta Edición. Pearson Educación.

(6) Maldonado C.E., Gómez Cruz N.A (2010) El Mundo de las Ciencias de la Complejidad un Estado del Arte. Universidad Colegio Mayor de Nuestra Señora del Rosario. Bogotá: Editorial Universidad del Rosario.

(7) Moreno J., Parra F., Huérfano R., Surez C. (2016) Modelo de Encriptacion Simetrica Basada en Atractores Caóticos. Revista Ingenieria. Universidad Distrital Francisco José de Caldas

(8) Levy D. (1994) Chaos Theory and Strategy:Theory, Application and Managerial Implications. Strategic Management Journal, Vol. 15

(9) Rodríguez Zoya, L. G. (2010). Complejidad y organización: Aportes del pensamiento complejo y la metodología de simulación de organizaciones sociales SocLab. VI Jornadas de Sociología de la UNLP, 9 y 10 de diciembre de 2010, La Plata, Argentina. En Memoria Académica.

(10) Pidal Gonzalez M.J. (2009). La Teoría del Caos en las Organizaciones. Cuadernos Unimetanos.

(11) Salazar Duque, O. (2017). Mirada de la gestión moderna desde la teoría del caos y la transdisciplina. Universidad \& Empresa, 19(33), 137-161. Doi: http:// dx.doi.org/10.12804/revistas.urosario.edu. co/empresa/a.5234 